\title{
The Contingent UnKNOWABILITY OF FACTS AND ITS RELATION WITH INFORMAL, EPISTEMOLOGICAL CONTEXTS
}

\author{
STANLEy KREITER BEZERRA MEDEIROS
}

\begin{abstract}
This paper focuses on elements that are involved in a specific type of judgment, namely, those involving facts that, in virtue of contingent reasons, are out of our epistemic reach. Its goal is to propose a philosophical explanation about why we, in informal contexts, take some facts as contingently unknowable. In order to accomplish that goal, we develop a theory that defines contingently unknowable facts in a very specific way. We establish three clauses that are jointly necessary and sufficient — so we argue — for taking an arbitrary fact as contingently unknowable. In a variety of contexts, this strategy has the potential of reducing efforts in an epistemological analysis of this particular type of unknowability.
\end{abstract}

Keywords: Necessary unknowability; contingent unknowability; evidence; contingency counterfactuals.

\section{Introduction}

The subject of unknowability is of great importance to human beings. Of course, if knowledge of our cognitive capacities is something that we should dedicate ourselves to, why not doing the same to our epistemic limitations? Knowing about our ignorance might be as useful as knowing about our creative capabilities, our eagerness to new discoveries and the possibilities they make available. While investigating our epistemic deficiencies, we might even reach fruitful conclusions not only about things that we are not able to know or accomplish, but also things that we shouldn't accomplish at all (due to our, say, "lack of wisdom" regarding the consequences of our actions). Another important reason for the inquiry on unknowability: knowing our ignorance is something that can help us understand our own role in this universe, our own place as intelligent beings (if there is any).

This paper will focus on the notion of "contingent unknowability". Not only does it presuppose that unknowable facts do exist, but also that there are different categories of such unknowable facts - the contingent ones are among those. The aim of this paper is not to demonstrate the existence of contingent unknowable facts. Instead, our goal is to propose a theory that philosophically explains why we, in informal contexts, take some facts as contingently unknowable. Therefore, this paper focuses on elements that are involved in a specific type of judgment, namely, those involving facts that, in virtue of contingent reasons, are out of our epistemic reach.

Principia 21(1): 61-76 (2017).

Published by NEL — Epistemology and Logic Research Group, Federal University of Santa Catarina (UFSC), Brazil. 
Our goal is to show why, in informal (and hence non-philosophical) contexts, reasoners might approximate trivial, contingently unknowable knowledge items to non-trivial (skeptical or "semi-skeptical") ones — as we call them. Though not a refutation of skepticism, nor an attempt to deal with the skeptical challenge in itself, we suspect that our specific treatment of contingent unknowability - that is, our use of a tripartite definition and a counterfactual approach - helps to explain, in a clear way, an expected attitude of such reasoners towards these contingent unknowable facts: the attitude of "not knowing it, not caring too much about it".

In informal (non-philosophical) contexts, the act of displaying relatively the same amount of attention to trivial and non-trivial unknowable facts by reasoners might be explained, perhaps, by the idea that those facts share the characteristics of being contingently unknowable, in the specific sense captured by our definition. This might be seen as a completely trivial observation in epistemology, so to speak. Granted, if we lack supporting evidence for believing $P$, we find ourselves in a bad epistemic position with respect to it (and this is due to contingent circumstances). In fact, there is nothing new to that.

However, this fact in epistemology does not explain why, in informal contexts, we might expect the same kind of epistemic judgment to apparently so different types of contingently unknowable facts. What we will try to do - and apparently, what hasn't been done in epistemology yet - is attempting to capture this type of epistemic attitude, that is, one that puts together (or at least approximates) facts expressed by sentences like "I'm not a brain in a vat", "what will happen to our universe trillions of years ahead" or "Julius Caesar's heart stopped beating at time $x$ of day $y$ " etc. Another important aspect to be mentioned is the fact that, while Nozick's (1981) counterfactual approach defines knowledge counterfactually, it doesn't focus on contingent ignorance. In truth, the relation between contingent unknowability and counterfactual epistemology is a rather unexplored subject in mainstream epistemology - though counterfactual epistemology is itself an important program of it (Hendricks 2006). The present work is an attempt to initiate such an investigation.

Granted, what we will offer is an explanation of a hypothetical practice made by hypothetical epistemic agents in hypothetical informal contexts. But we believe that not even this has been efficiently done in mainstream epistemology. By assuming that the practice of approximating trivial to non-trivial contingently unknowable facts really takes place in informal contexts, we will try, in turn, to show what makes them so similar to the "eyes" of those agents, that is, what makes them apparently so close regarding their epistemic status. And we will do this by showing successful applications of our counterfactually inspired definition to five formulated, different cases involving contingently unknowable facts.

This paper is divided in six sections. Section 2 discusses necessary unknowability. Section 3 is dedicated to the formulation of cases involving contingent unknowability 
as well as an informal discussion on the contingent limits to our knowledge as finite intelligent beings. In section 4, the theory of contingent unknowability is briefly exposed. The discussion of the theory will be left to section 5 . We conclude in section 6 , then, by presenting some arguments of why such an approach to contingent unknowability might be useful to epistemology - especially to the discussion on the unknowability of some skeptical hypotheses.

\section{Necessary limits to knowledge: necessary unknowability}

Lack of omniscience is a reality for concrete, human epistemic agents. This means there are some facts that are unknown to us. But we can go further. It is possible for us to conceive something stronger: some facts are simply unknowable - out of our epistemic reach. Let's call them "unknowable facts". Such unknowable items might have different causes and, because of that, it is important to make a distinction between "necessary" and "contingent" unknowability.

Necessary (or demonstrable) unknowability happens when the cause of the unknowability is necessary or inevitable. As Rescher shows (2009, p.3), this idea "admits of three construals:

1. logical unknowability - demonstrable on the basis of abstract considerations of epistemic logic.

2. conceptual unknowability - demonstrable on the basis of an analysis of the salient concept and ideas at issue.

3. in-principle unknowability - demonstrable on the basis of the fundamental principles that delineate some area of inquiry or deliberation."

Fitch demonstrates (1963, pp.138-9) two interesting theorems on logical unknowability. The theorems stated two powerful things:

- for each agent who is not omniscient, there is a true proposition which that agent cannot know;

- if there is some true proposition which nobody knows (or has known or will know) to be true, then there is a true proposition which nobody can know to be true.

This type of unknowability is logical since it can be demonstrated purely on logical grounds. Suppose that $F$ represents the fact that there were 600 Spartans at the battle of Thermopylae, instead of 300. Let's assume that an epistemic agent, $S$, doesn't know that fact. Now observe that, if $S$ doesn't know $F$, it is logically impossible for $S$ to know the following fact:

Principia 21(1): 61-76 (2017). 
$F_{2}:$ (1) There were 600 Spartans at the battle of Thermopylae and (2) $S$ (himself) doesn't know that fact. ${ }^{1}$

Note that $F_{2}$ is a conjunction of two facts: (1) there were 600 Spartans at the battle of Thermopylae and (2) $S$ doesn't know there were 600 Spartans at the battle of Thermopylae. If $S$ were to know $F_{2}$, by distribution of conjunction he would have to know (1), which is being denied by the premise that $S$ doesn't know $F$. Besides, knowing (2) would also be incompatible with the hypothesis: for $S$ to know that he doesn't know $F$, it would be necessary (for $S$ ) to recognize $F$ as a fact in the first place - which is being denied by our initial assumption.

Therefore, there is a logical contradiction involved in the assumption that $S$ could possibly know $F_{2}$. This means that a proof by reductio ad absurdum is well succeeded in establishing the impossibility for $S$ to know $F_{2}$ - a logically unknowable fact, given that $S$ doesn't know $F$. Rescher (2009, p.3) points out that "[ [.. ] it is logically demonstrable that one cannot know that such-and-such a particular fact is among those one does not know".

There is one important aspect to be highlighted about this particular kind of unknowability: it can be stated as a logical principle or theorem: "If $S$ doesn't know $F$, then the following fact is unknowable: $F$ is a fact and $S$ doesn't know $F$ ". As a general theorem, it is totally indifferent about who $S$ is or what the fact $F$ stands for.

But in the case of conceptual unknowability, things are a bit different. Here, the meaning is important indeed. It is called "conceptual" because there is need for a concept, for the meaning of a particular expression. However, as soon as the concept is understood, unknowability follows necessarily. For example, suppose that $A$ is an idea that nobody did, or does or will consider. By definition, this type of idea can't be known by anybody. This is prohibited by the very concept of an idea that "hasn't been and won't be considered by anybody". The same applies to events. If $E$ is both (1) an event that was not observed and (2) that left no trace of its occurrence that is, an event whose traces haven't and won't be observed - , it is, by definition, unknowable.

The third type is controversial and interesting at the same time. There is no objective way of deciding when the Portuguese word república was used for the first time in that language. ${ }^{2}$ What do we mean by "using it", in the first place? Do we mean "using it" by "pronouncing it" for the first time? But as we all know, the Portuguese language comes from vulgar Latin; the word república comes from two Latin words, namely, res and publica. So we have reasons to think that republica occurred before the Portuguese language but also after the period of vulgar Latin — and in different locations as well, such as Spain, Italy, Great Britain etc. Which came first, the Portuguese word república or the Spanish word republica? Should we include location or context, then? What about specific cultural aspects? But again, do we have objective 
criteria for such choices? Since there is no objective way of deciding when a particular instance counts as the first and unique occurrence of a word in a language, there is no way of knowing when that particular word was used for the first time in that same language. Therefore, the first occurrence of the Portuguese word república is in principle unknowable to us.

\section{Contingent limits to knowledge: contingent unknowability}

In our daily lives, we constantly make judgments that presuppose a certain conception of contingent unknowability. This is not unusual since there are some facts that are bound to be unknowable to us in virtue of contingent reasons. In other words, that there are contingently unknowable facts is a trivial observation in epistemology. Usually, contingently unknowable facts can be associated with questions that we tend to conceive as unanswerable. To illustrate that, let's take a look at the following questions:

(1) How many cats were there in the world at the time of Socrates' execution? Was there any cat present in that tragic occasion?

(2) Were Socrates and Jesus enlightened aliens from a much more advanced society? Is there any such being among us right now?

(3) Are we prisoners of the Matrix?

(4) Is there any intelligent life form in those regions of the universe that are bound to be unobservable by us?

As we can see, it is very hard to provide a satisfactory answer to any of these questions. Nonetheless, from (2)-(4), each one is a type of question that can be answered with a simple "yes" or "no" - the same applies for the second part of (1). The first part of (1) also has a simple answer: a finite number. There is no logical impossibility involved in providing an answer to any of the questions above. Since there is no such impossibility, we all can conjecture possible answers - with only one inconvenient, though: our answers will probably lack supporting evidence. Because of this - and perhaps with the exception of philosophy or the sciences - not many people will care answering these anyway. We simply call them unanswerable (not in a philosophical or scientific sense, perhaps, but in an informal, common sense which is just enough to our practical purposes).

These are the types of questions that we will call "contingent unanswerable": and that's because our ineptitude in answering them stems not from any logical, necessary impediment - as we have seen with necessary unknowability. Rather, it stems from a contingent, accidental, lack of evidence - due to a contingent, accidental configuration of the actual state of affairs.

Principia 21(1): 61-76 (2017). 
These contingently unanswerable questions are directly linked with what we shall call contingently unknowable facts. These are very common indeed, and are present in informal judgments of the type "we will never know that ...", "we couldn't possibly know that" and the like.

There is an interesting remark to be made about that. Thought it is indeed a trivial observation in epistemology that those questions might be contingently unanswerable, the reason why we, in informal situations, might expect similar kinds of epistemic judgments to apparently so different types of questions, or facts, is not properly explained. Considering situations (or contexts) in which some epistemic agents will regard all of these to be simply "unknowable" — in spite of clear differences in their status, rigorously speaking - , we will try to make a point on what makes them look so similar to these agents. We will try to show which shared characteristics those facts have in common so that, in such informal contexts, they all can be regarded as contingently unknowable.

Now, to illustrate the idea, let's consider five different cases:

(I) The "alien's" case. Suppose that a very advanced alien civilization, while interested in studying the human species, sent one of them - let's call him " $X$ " — to planet Earth five thousand years ago. In virtue of their technology, these aliens can modify their body and DNA so that they look exactly with the ones shared by ordinary human beings. Thus, the alien they sent has virtually all characteristics that an ordinary human has, with just one important exception: he's not human. Now suppose that an ordinary human agent, say, Smith (who has become a friend of our alien observer), doesn't know that. Considering that Smith is not the first friend $X$ has made - remember, our alien friend has been here since 5000 years ago - , there is reasonable evidence to suppose that Smith will never know that his friend is an alien. This, of course, is not to say that the fact that $X$ is an alien is necessary unknowable or that knowing it is logically impossible. In truth, the possibility of Smith coming to know this fact still exists, though very unlikely. The point is that, in an informal context, it is reasonable to assume that the fact that $X$ is an alien is unknowable to Smith in virtue of the actual state of affairs in which Smith is in - and which, of course, is contingent. In other words, the fact that $X$ is an alien is contingently unknowable to Smith.

(II) The "Wait, please!" case. For this case, let's suppose two agents: Smith and Jones. Smith is Jones's father. For many years, he has kept secret from Jones the fact that he, Smith, was a Nazi SS officer during World War II. Now, in his dying bed, Smith tries to tell Jones the whole truth. Unfortunately, the only thing he manages to say before he passes away is that he had a secret to share, nothing more. Assuming that his testimony would be the only remaining evidence of this particular part of his past, Jones lost the only chance of knowing this particular secret about his father.

Principia 21(1): 61-76 (2017). 
Thus, similarly, it is reasonable to say that the fact that Smith was a Nazi SS officer becomes contingently unknowable to Jones.

(III) The "precise numbers" case. Assuming that Socrates, the philosopher, was really judged and executed in the year 399 BCE, we might wonder: what was the precise number of individuals that attended both trial and execution? Or, we can conjecture something much more general: how many hound dogs were alive in the world at that time? Again, these are the types of question to which we can answer with a simple, definite number. The answer, in turn, would have the following format: "there were ' $x$ ' individuals at Socrates' trial"; "there were ' $x$ ' hound dogs alive in the world when Socrates was executed". The " $x$ " is a marker for a definite number, which is unknown to us. We can go further and argue, of course, that " $x$ " is not just unknown to us; given the current conditions and the actual state of affairs, it is reasonable enough to infer that " $x$ " is unknowable to us - in that contingent, informal sense we've been discussing.

(IV) The "Matrix" case. In the 1999 science fiction Hollywood movie, Neo was a prisoner of a virtual reality called "The Matrix". As the film suggests, that "mental" prison was inescapable unless the prisoner was freed by someone who was already freed and hence knew about the Matrix. ${ }^{3}$ In that respect, the fact that Neo was a prisoner of the Matrix was contingently unknowable to Neo, while he was himself a prisoner. It is possible for us to enlarge the context and conjecture about other skeptical hypotheses: "brains in vats", "malicious demons", "permanent dreaming" etc. All of them have the same effect in the epistemological discussion. We will argue that all of these are unknowable in that contingent, informal sense we've been discussing.

(V) The "Universe's future" case. There are at least three important scenarios to be considered when we think about the distant future of the universe and its end: (i) the big rip, (ii) big chill and (iii) big crunch. In the first one, expansion will accelerate until the very forces that bound atoms together won't be enough to hold them anymore. The result will be the complete disintegration of stars, our bodies etc. In the second scenario, the expansion continues forever, but won't be sufficient to rip ordinary matter apart. After trillions of years, all galaxies, stars, black holes will disappear. The universe will be cold, dark, empty and eventually fall into complete oblivion. In the third and last scenario, gravity will stop expansion and make the universe to contract. Eventually, the universe will collapse in itself. Which one is going to be? No one can answer that with certainty. Even if an answer is provided and that answer is backed by considerable supporting evidence, this won't give us certainty. Since there is also good supporting evidence to think that our species won't probably exist anymore when that happens, we can't decisively eliminate the possibility that something else might happen (after we are gone) that can alter the result we had reason to expect. In this sense, what will really happen with the universe trillions of 
trillions of years ahead is a fact that is bound to be contingent unknowable to us, given the actual state of affairs - which includes the current technological status of our species and the scientific knowledge that we have about the universe.

So, all of these five cases are taking into consideration a notion that we are calling "contingent unknowability". All the facts presented in each one of the five cases are, in a way or another, out of our cognitive reach right now - and might stay that way forever. Our intention, of course, is not to prove that. We already behave ourselves and reason about them in a way that clearly shows our inclination to judge them as contingently unknowable.

Our goal here is to propose a theory that philosophically explains what is involved in this type of informal judgment. That is, why do we make this type of judgment at all? Which are the elements that together constitute a contingent unknowable fact? So, how can we determine when some particular fact - say, F — can be considered contingently unknowable? In the sections to come, we will answer these questions by exposing the theory and showing, by applying it to the five formulated cases above, how it works.

\section{A theory for the contingent unknowability of facts}

One of the main aspects of contingent unknowability is its close relation with counterfactual conditions. For instance, “if Morpheus hadn't freed Neo, he would still be a prisoner of the Matrix", "if I had a time machine, I would know the precise number of individuals that personally watched Socrates' trial". The counterfactual element is important here because it demonstrates that there is no necessary, logical impediment to other scenarios that would be capable of changing the epistemological status of a fact - that is, from "unknown" to "known", or "unknowable" to "knowable". Given its importance to our theory, we shall define it like this:

Definition CC [contingency counterfactual]. Let $F$ be any fact and $S$ any epistemic agent that doesn't know $F$. Let C-FACT be any counterfactual and $C$ a non-empty set of conditions of C-FACT. We say that C-FACT is a contingency counterfactual to $F$, relative to $S$ if, and only if, it has the following form: if the set $C$ of conditions were satisfied, $F$ would be known to $S$.

If, as examples, we use the five cases of the previous section coupled with the definition above, we can create a contingency counterfactual related to each one of them:

Case 1: "If Smith's alien friend had taken him to his alien world and shown the truth about him, Smith would have known that his friend was an alien."

Principia 21(1): 61-76 (2017). 
Case 2: "If Smith had told Jones the true story about himself before passing away, Jones would have known that his father was a Nazi SS Officer in World War II."

Case 3: "If we had a time machine, we would know the precise number of individuals that were present at Socrates' trial."

Case 4: "if Morpheus freed Neo, Neo would know that he was a prisoner of the Matrix."

Case 5: "If we became immortal, indestructible and had the power of creating other universes, we could observe, from another universe, what would happen to our own universe trillions of years ahead."

As we can see, each one of these counterfactuals falls under definition CC in relation to cases $I-V$, respectively. It's worth noting that the number of possible counterfactuals we can create to each case is infinite. The important point, though, is the mere possibility of associating at least one contingency counterfactual to a fact. This key ingredient, despite not being enough, is crucial in identifying a contingently unknowable fact.

With that in mind, we now present a precise definition for a contingently unknowable fact:

Definition CUF [contingently unknowable fact]. Let $F$ be any fact, $S$ be any epistemic agent and C-FACT be a contingency counterfactual, as presented by definition CC. We say that $F$ is contingently unknowable to $S$, in a given moment $t$, if and only if:

a. $S$ doesn't know $F$, in time $t$;

b. in the epistemic context in which $S$ is in, $S$ doesn't have access to supporting evidence for establishing or confirming $F$, and the probability of getting it is considered very low.

c. C-FACT is a contingency counterfactual for $F$, relative to $S$.

We argue that, if there is a success in demonstrating the connexion between $a, b$ and $c$ to any $F$, then that fact can be considered contingently unknowable. In the next section, we apply definition CUF to the cases presented in the previous section. The purpose is to show, through these cases, that the elements of definition CUF are precisely the ones involved in judgments of contingent unknowability - that is, in informal judgments that we usually make, or would make, on those cases.

\section{Applying the theory and discussing its elements}

Let's begin the application of the theory by considering case 1 . Assuming it is a fact that $X$ is an alien, we have that:

Principia 21(1): 61-76 (2017). 
a. Smith doesn't know that $X$ is an alien;

b. In the epistemic context in which Smith is in, Smith doesn't have access to supporting evidence for establishing or confirming that $X$ is an alien, and the probability of getting it is considered very low.

c. If $X$ wanted to reveal his secret to Smith, Smith would know $X$ is an alien.

The first element seems to be straightforward. Of course, in an informal context, if we assume that some fact is unknowable, that's because it is taken as unknown in the first place. If we say that nobody will ever know what is going to happen trillions of years ahead, we automatically assume that nobody currently knows what is going to happen trillions of years ahead. As we can see, clause "a" is not sensitive to the context in which the epistemic agent is in. In a way, it is more like a logical requirement. Let's call it "the logical requirement", then.

That, of course, is not the case with clause "b", which is context sensitive. "b" represents what we shall call the "evidential requirement". This clause is perhaps the most philosophically problematic of all three. It assumes that not only do knowledge ascriptions depend on context, but they also seem to push - in virtue of this very context - a certain arbitrary consensus on probability attribution. The discussion about the philosophical grounds for clause "b" - as well as its philosophical justification - has a place on its own, so we shall leave it to another occasion. For now, we're just applying the theory and showing how it works. ${ }^{4}$

The final element expressed in clause "c" - the counterfactual one - stands for the fact that $F$ is not unknowable "in-principle", nor conceptually nor logically. It guarantees that $\mathrm{F}$ stays contingent by recognizing the possibility that different, counterfactual scenarios could alter its epistemic status.

Case 1 illustrates, perhaps, a "semi-skeptical" scenario. We use the term "semiskeptical" because it casts doubts on basic assumptions - like "that is a real human person" - without calling into question our very reality, as does case IV. Cases II and III are not skeptical in this sense; they rely on very plausible scenarios that are not very different from what we take to be our own actual state of affairs. As we can see, it is also possible to apply definition CUF to both cases, respectively:

(Case II)

a. Jones doesn't know his father, Smith, was a Nazi SS Officer during World War II.

b. in the epistemic context in which Jones is in, Jones doesn't have access to supporting evidence for establishing or confirming that Smith was a Nazi SS Officer during World War II, and the probability of getting it is considered very low. 
c. If Smith had been able to tell his secret to Jones before passing away, Jones would know that Smith was a Nazi SS Officer during World War II.

Thus, according to definition CUF, since "a", "b" and "c" all apply, the fact that Smith was a Nazi SS Officer during World War II is contingently unknowable to Jones.

(Case III)

a. We don't know the precise number of individuals that were present at Socrates' trial.

b. in the epistemic context in which we are in, we don't have access to supporting evidence for establishing or confirming the precise number of individuals that were present at Socrates' trial, and the probability of getting it is considered very low.

c. If we had a time machine, we would know the precise number of individuals that were present at Socrates' trial.

Then, since "a", "b" and "c" all apply, the number of interest is contingently unknowable to us.

Case IV is special in the sense that it is skeptical. It casts doubts on very basic assumptions - like our very reality. Skeptical scenarios might include, as Dretske puts it, "heavyweight propositions" (Dretske 2014a; 2014b). We can show, however, that our definition can be successfully applied to case IV as well:

a. Neo doesn't know he is a prisoner of the Matrix.

b. In the epistemic context in which Neo is in, he doesn't have access to supporting evidence for establishing or confirming if he is a prisoner of the Matrix, and the probability of getting it is considered very low.

c. If Morpheus freed Neo, Neo would know that he was a prisoner of the Matrix.

Hence, since "a", "b" and "c" all apply, that he himself is a prisoner of the Matrix is a contingently unknowable fact to Neo.

This case deserves special attention, though. Observe that the skeptical hypothesis, or scenario, can be conjectured ad infinitum. Suppose that Neo was indeed a prisoner of the Matrix and that Morpheus really set him free from it. In the alleged "real world", Neo might wonder, again, if he is a prisoner of something like the Matrix - say, Cavetrix. The new Cavetrix scenario constitutes a new possibility that can't just be simply ignored. On the other hand, there is no logical impediment in applying definition CUF again and again. In other words: despite being possible to conjecture an infinite number of skeptical scenarios in order to undermine knowledge about any fact, it is also possible to apply definition CUF with the purpose of showing these 
facts are not necessarily unknowable; as in the previous cases, the application of CUF shows that they are contingently — not necessarily — unknowable.

So, we argue that definition CUF might explain why, in an informal (non-philosophical) contexts, these agents might not be tempted to embrace total skepticism: CUF guarantees that the fact of interest remains, at most, contingently unknowable like many other trivial facts. In our view, what CUF does is to put, at least in informal (non-philosophical) contexts, "skeptical-type" ignorance very close to other trivial types of ignorance - and by "trivial" we mean the ones that are not philosophically problematic, as is the case of ignorance about facts regarding the future.

CUF indeed brings skeptical and non-skeptical scenarios closer in this respect, that is, in their contingency and susceptibility to counterfactual conditions. Though apparently trivial, that is an interesting phenomenon since, in informal epistemic contexts, we might expect agents to show similar epistemic attitudes toward the following questions:

- How do you know, with certainty, that your best friend is not a disguised alien — or a cyborg, perhaps?

- How do you know, for sure, that your grandfather was not a SS officer?

- How do you know, for sure, that there wasn't any cat present during Socrates' execution?

- How do you know, for sure, you're not a prisoner of the Matrix?

There is one more important aspect to be considered, though. "To put skepticaltype ignorance very close to other trivial types of ignorance" doesn't mean to fuse them in one and the same object. This would be to oversimplify the differences between skeptical and non-skeptical hypotheses. As we all know, the denial of any nontrivial, skeptical hypothesis has a different epistemological status from those that are trivial, mundane ones. This happens because the skeptical challenge is a problem about generalized ignorance, which stems from the notion of global underdetermination:
A hypothesis is globally underdetermined if there are two possible worlds such that one of the worlds assigns the truth-value true to the hypothesis while the other assigns false in such a way that the evidence received by the agent remains the same forever independently of which of the two worlds is the actual world. (Kelly 1996, p.17)

In case IV - the Matrix case - , though prisoner of the Matrix, Neo receives the same evidence that he would get if he wasn't such a prisoner. The Matrix world assigns false to the hypothesis that "Neo is a prisoner of the Matrix" (deceiving him), while the real worlds assigns true to it. In terms of evidence received, it makes no dif- 
ference to Neo. Thus, to him, the hypothesis "I'm a prisoner of the Matrix" is globally, not locally, underdetermined; it is a problem of global, not local ignorance.

This detail is important because there is a relevant objection regarding the strategy of taking skeptical hypotheses as describing contingently unknowable facts. In considering the fact expressed by a skeptical proposition as "contingently unknowable", we might be tempted to take such proposition simply as "the one to which we don't have supporting evidence"; and hence, we might be turning the skeptical challenge into a problem about localized ignorance - that is, ignorance of one specific proposition - , when this is clearly not the case.

But again, observe that the lack of evidence - that is, the evidential requirement - is just one of CUF's elements. In order do to its job, namely, to properly characterize contingently unknowable facts (and hence the propositions that describe them), CUF needs all its elements working together. In other words, the counterfactual element - precisely defined in CC - can't be separated from the analysis. But again, the idea is not to solve the skeptical challenge or to prove the falsity of skeptical hypotheses. Rather, the idea is just to show that they, skeptical hypotheses, have the property of sharing a characteristic with non-skeptical ones: the property of being contingently unknowable, in the precise sense expressed by definitions CC and CUF. And we think that, precisely because they have that property, they seem so similar to trivial hypotheses in informal contexts. That's why, in informal epistemic contexts, we might expect similar epistemic judgments about Matrix type scenarios, brains in vats, alien friends, distant past and future events and so on. That being said, let's now turn to our last case: contingent ignorance about the future.

Case $\mathrm{V}$ represents a typical case of ignorance about the future or, in other words, about a fact regarding the future. The type of ignorance Case V illustrates is, perhaps, as common in scientific or philosophical contexts as it is in informal ones. It doesn't matter if our epistemic standards are high or low, ignorance about the distant future seems not to be a big of a problem: we simply admit our ignorance on the matter. However, through definition CUF our conception about contingent unknowability assures that these types of facts similarly remain, at most, contingently unknowable. Let's use Case V as example:

a. We don't know what precisely is going to happen to our universe, trillions of years ahead.

b. In the epistemic context in which we are in, we don't have access to supporting evidence for establishing or confirming what precisely is going to happen to our universe, trillions of years ahead, and the probability of getting this evidence is considered [very?] low.

c. If we developed our science and technology so that we became immortal, indestructible and capable of creating universes, we would be able to move into 
another universe and would, then, be able to see what is going to happen to our original universe, trillions of years ahead.

As crazy or unlikely clause "c" might seem, when put together with "a" and "b", it guarantees the contingency of our ignorance about the distant future of our universe. Despite very unlikely, it is not necessarily impossible for us to become god-like creatures. Thus, by "a", "b" and "c", what precisely will happen to our universe, trillions of years ahead, is a contingently unknowable fact to us.

That concludes the application of the theory to the five cases we've been discussing. The theory, hence, is based on a particular definition of contingently unknowable fact. This definition establishes three conditions that together constitute, we argue, necessary and sufficient conditions for taking a particular fact as contingently unknowable. They are, thus, essential constituent elements of informal judgments involving contingent ignorance about facts.

\section{Conclusion}

While investigating the boundaries of our own knowledge, we find that there are at least two types of unknowable facts: the necessary and the contingent ones. Necessary facts can be devised in three categories: logical, conceptual and in-principle each one corresponding to a specific case of necessary unknowability. These, in turn, are very different from the contingently unknowable ones, which depend on the contingent arrangement of our actual state of affairs. Our theory for this particular type of unknowability establishes three clauses that, together, constitute necessary and sufficient conditions for taking an arbitrary fact to be contingently unknowable.

Each clause of the definition relates to a necessary element in any judgment involving contingent unknowability about a particular fact, and these are: ignorance regarding the fact (logical requirement), contingent lack of information for knowing the fact (evidential requirement) and a contingency counterfactual related to the fact (counterfactual requirement). If these three requirements can be successfully applied to any fact, say, $F$, then $F$ can be considered contingently unknowable.

This strategy captures what is, in our opinion, an already common practice to people when reasoning about those things in informal contexts. If asked to emit judgments on the "facts" displayed in cases I-V, as well as the possible problems associated with those judgments, an agent would probably end up with answers like "we will never know that for sure", "we couldn't know that" etc. It is precisely to these types of epistemic judgments that our theory applies. It describes a specific practice of emitting epistemic judgments on a particular class of facts, in a particular type of context - that is, an informal, non philosophical one. In our view, the logical, evidential and 
counterfactual requirements are those which are necessary and sufficient to explain epistemic judgments in informal contexts.

Our approach to contingent unknowability has some interesting features. First, it tries to recognize the weight and importance of the skeptical challenge by granting a certain degree of ignorance to an infinite number of trivial (and non-trivial) facts without, however, embracing skepticism completely. In other words: for a vast variety of facts, our ignorance about them is just contingent, not necessary - assuming an informal epistemic context, of course. Among other things, this ignorance might be about: strong skeptical hypotheses, weak skeptical hypotheses, unknown trivial (nonskeptical) facts and unknown facts about the future.

At the same time, our theory not only takes into account qualitative and quantitative aspects of our ignorance about a vast variety of facts; it also shows that many of these facts share one common characteristics: being contingently unknowable. In a variety of contexts - in informal ones, especially — this might reduce efforts in the analysis of their epistemic status.

Second, our definition of contingent unknowable fact tries to be as clear as possible so that any doubt regarding its elements can be precisely directed. One example is clause $b$, the evidential clause. It appeals to a philosophically problematic perspective that places evidential quality, its access and probability attribution subject to context variations. As we said before, this clause renders a discussion on its own and will be addressed in another work.

\section{References}

Dretske, F. 2014a. Is knowledge closed under known entailment? The case against closure. In: M. Steup (ed.) Contemporary debates in epistemology. Malden: Blackwell. 2014b. Reply to Hawthorne. In: M. Steup (ed.) Contemporary debates in epistemology. Malden: Blackwell.

Fitch, F. B. 1963. A logic analysis of some value concepts. The Journal of Symbolic Logic 28(2): 135-142.

Hendricks, V. F. 2007. Mainstream and formal epistemology. New York: Cambridge University Press.

Kelly, K. T. 1996. The logic of reliable inquiry. New York: Oxford University Press.

Nozick, R. 1981. Philosophical explanations. Massachusetts: Harvard University Press.

Rescher, N. 2005. Epistemic logic: a survey for the logic of knowledge. Pittsburgh: University of Pittsburgh Press.

- 2009. Unknowability: an inquiry into the limits of knowledge. Lanham: Lexington Books.

Stanley Kreiter Bezerra Medeiros Instituto Federal do Rio Grande do Norte Campus Ceará-Mirim

Principia 21(1): 61-76 (2017). 
BRAZIL

stanley.medeiros@ifrn.edu.br

\section{Notes}

${ }^{1}$ It's assumed, of course, that a conjunction of two facts is itself a fact.

${ }^{2}$ Here we take the liberty of providing our own adaptation of the example offered by Rescher (2009, p.3).

3 To gain freedom, the subject would have to take the infamous "red pill"...

${ }^{4}$ Although it's worth noting that the aim of the theory is, through the use this particular conception on contingently unknowable facts, to describe an already common practice between epistemic agents in informal contexts.

Principia 21(1): 61-76 (2017). 